\title{
Experiences and Lessons Learnt from the HIV Treat All Pilot Phase Implementation in Zimbabwe
}

\author{
Richard Makurumidze (iD) ${ }^{1-3}$ \\ 'Faculty of Medicine and Health Sciences, \\ University of Zimbabwe, Harare, \\ Zimbabwe; ${ }^{2}$ Department of Clinical \\ Sciences, Institute of Tropical Medicine, \\ Antwerp, Belgium; ${ }^{3}$ Gerontology, Faculty \\ of Medicine \& Pharmacy, Vrije \\ Universiteit Brussel (VUB), Brussels, \\ Belgium
}

\begin{abstract}
Zimbabwe adopted the Treat All recommendations and started to implement them nationwide in 2017. Before launch, Treat All was piloted in nine districts. The sharing of implementation experiences and knowledge gained will significantly contribute to the implementation success in settings where Treat All still needs to be rolled out. We report on experiences and lessons learnt from the implementation of Treat All during the pilot phase in Zimbabwe. Coordination and well-structured engagement plans with the districts led to the successful implementation of the Treat All pilot. The established technical working groups offered standardisation and a platform for the exchange of experience between the implementing partners and the Ministry of Health and Child Care. Training and capacity building of the healthcare workers through mentoring, support and supervision, and the provision of job aides were necessary to equip them with the required skills. Community knowledge, commitment and support were critical to the successful implementation of the guidelines. Health facilities preparedness was of great importance. This enabled the health facilities to develop mitigating strategies at the local level using existing resources. Studies examining how patient outcomes will progress under Treat All are pertinent.
\end{abstract}

Keywords: HIV, antiretroviral therapy, Treat All, sub-Saharan Africa

\section{Introduction}

The World Health Organization (WHO) in 2016 recommended initiating antiretroviral therapy in all HIV infected patients regardless of clinical or immunological criteria ie Treat All. ${ }^{1}$ Studies have shown clinical benefits of starting antiretroviral therapy (ART) early. ${ }^{2,3}$ Thirty-five countries, accounting for more than $90 \%$ of new HIV infections, have been designated Fast-track countries requiring intensified action. The Fast-track targets are aimed at achieving: 90\% of the people living with HIV (PLHIV) know their HIV status, $90 \%$ of the PLHIV who know their HIV-positive status are accessing treatment and $90 \%$ of the people receiving treatment have a suppressed viral load by $2020 .{ }^{4}$

Countries, including those in Eastern and Southern Africa, are fast adopting the WHO Treat All recommendation to enable them to reach the 2020 Fast-track targets. ${ }^{5}$ Results from countries that started the implementation of Treat All early on are encouraging. ${ }^{6}$ This indeed shows that with the scale-up of HIV Treat All, if coupled with additional measures to retain HIV-positive persons newly initiated on treatment, the 2020 Fast-track targets are achievable. However, implementing and optimising Treat All, including strategies to address the anticipated challenges in a resource-constrained setting, is yet unknown. ${ }^{7}$

Zimbabwe is among these Fast-track countries and has since adopted the Treat All recommendations. Treat All is currently being implemented countrywide. The
Correspondence: Richard Makurumidze Faculty of Medicine and Health Sciences, University of Zimbabwe, Harare,

Zimbabwe

Tel +263772759296

Email rmakurumidze@ext.itg.be 
Treat All guidelines were launched on World AIDS Day, the $1^{\text {st }}$ of December in $2016 .{ }^{8}$ Before the launch, Treat All was piloted in nine districts (Chipinge, Bulilima, Gwanda, Harare, Mangwe, Makoni, Mazowe, Mutasa and Mutare) of Zimbabwe. The pilot was for six months and started in June 2016, and by August of the same year, all the nine districts had started implementation. Sharing implementation experiences and lessons learnt will go a long way towards implementation success in settings yet to scale up Treat All. We report the experiences and lessons learnt from the implementation of the HIV Treat All during the pilot phase in Zimbabwe.

\section{The HIV Treat All Pilot Phase Implementation Activities and Experiences}

\section{Coordination}

The pilot was mainly led and supported with the United States Presidential Emergency Plan for AIDS Relief (PEPFAR) funding through its two main implementation partners (International Training Education and Centre for Health - I-TECH and Organisation for Public Health Intervention Development - OPHID). The Ministry of Health and Child Care (MoHCC) provided the necessary support at every level of its structures to ensure the pilot's success. The MoHCC at the national level assisted with introducing the partners to the provincial and district structures for support, buy-in, and ownership of the pilot. A schedule illustrating key steps was developed for every district to facilitate the implementation.

The MoHCC led the adaption process of the new guidelines, resource mobilisation and revision of the monitoring and evaluation tools. To monitor implementation, PEPFAR partners set up four technical working groups (TWG); Training and capacity building, Linkage and referral of clients, Learning and documentation and Communications. These TWGs met regularly during the pilot to get feedback from partners, review and map the implementation way forward.

\section{Healthcare Workers Training and Capacity Building}

Before the start of the pilot, implementing partners conducted sensitisation and training meetings with healthcare workers and district health managers. The training and capacity building TWG led the process of developing a standard sensitisation and training package. The package covered the following topics: the 90-90-90 targets, the rationale of Treat All, clinical assessment of opportunistic infections, adherence counselling, antiretroviral therapy initiation and patient follow-up. To aid understanding among healthcare workers, the MoHCC and the supporting partners developed tools to support implementation. The following tools were developed: Operational and Service Delivery Manual (OSDM) for the Prevention, Care and Treatment of HIV in Zimbabwe; ${ }^{9}$ Consolidated HIV \& AIDS Job Aide; ${ }^{10}$ Frequently asked questions on Treat All $^{11}$ and HIV Retesting: Frequently asked questions. ${ }^{12}$ The OSDM manual was the primary document that provided the details on how to implement Treat All while the job aide was a desk tool for easy reference by healthcare workers. On top of the training and supporting tools, the healthcare workers were supported with on the job training, supervision and mentoring by mentors from MoHCC and partners.

\section{Mobilisation, Sensitisation and Awareness Campaigns for the Community}

The Communications TWG led the development of the Treat All Communication Strategy. The strategy aimed to develop knowledge and awareness on the Treat All implementation package and generate demand for services across the cascade of care (HIV testing, linkage to care, ART initiation, and viral load monitoring). One day meetings were conducted in all the districts to sensitise community structures, which included traditional and political leaders, community-based organisations and networks of people living with HIV. The focus of the meetings was to make community members understand that all populations and age groups were now eligible for treatment if HIV positive. Simplified materials were used in the sensitisation of these community cadres to aid understanding. During the meetings, concerns, myths and misconceptions about Treat All were identified and clarified.

The Treat All Communication Strategy included a standard slogan, "HIV Treatment; Get on. Stay on. Live on", which was translated to common local languages. A logo based on that theme was also designed for use on the various awareness campaign, information, education and communication materials. ${ }^{13}$ The strategy also contained various demand creation strategies and activities together with guidance on how to implement them. 


\section{Health Facility Readiness and Preparedness}

The healthcare workers were engaged to undertake a detailed review of health facility processes and identify site-level barriers that can affect the implementation of Treat All. The assessments were conducted using a detailed checklist which covered the following issues: availability and training of human resources, HIV testing services, linkage to care, ART initiation, retention in care, ART commodities and documentation. ${ }^{14}$ The assessment of ART commodities (HIV test kits, antiretroviral drugs, CD4 testing and viral load monitoring commodities) was meant to prevent stock-outs. Health facilities were trained to perform data-driven quantification working closely with the district laboratory and pharmacy departments. They were then supposed to use the findings from the site readiness and preparedness assessment to come up with actions to address the challenges and barriers identified.

\section{Patients Who Had Antiretroviral Therapy Deferred Due to Eligibility Criteria}

Some of the patients who had ART deferred due to eligibility criteria came alone for ART initiation upon hearing the change in guidelines through various community mobilisation, sensitisation and awareness campaigns modalities that were in place. There was also an active followup of these patients by health facilities. The process involved line listing these patients using the pre-ART and HIV testing registers. The line lists included the patient contact details. These patients were then tracked through short message service (SMS), phone calls and the use of community healthcare workers. Partners supported health facilities with airtime for SMS and making calls. In the early stage of the pilot, the number of new ART initiations surpassed the new positives identified because of these patients who had ART deferred due to eligibility criteria. ${ }^{15}$

\section{Antiretroviral Therapy Initiation Processes}

The coming of Treat All saw patients being started on ART without CD4 count and on the same-day. The patients underwent clinical and psychological readiness assessments before ART initiation. Clinical readiness was assessed mainly through history and physical examination. Healthcare workers were trained, mentored and supported in the screening and identifying common opportunistic infections (tuberculosis, cryptococcal meningitis and
Kaposi sarcoma). Clinically ready patients underwent psychological readiness assessment. The previous psychological preparedness (counselling sessions) had four sessions that covered basic HIV \& AIDS and adherence counselling. These would typically be conducted over a period of a week. The sessions were now compressed into a single day session to cater for patients initiating ART on the same-day. To a few who failed to absorb the information on the same-day, ART was deferred or started. Those who began ART without completing the counselling sessions were booked to come and finish the remaining sessions while already on ART. To improve linkage to care, the Linkage and referral of clients TWG developed a standard referral form for use in referring clients from one service delivery point to another. The referral form had a tear-off section to allow feedback and confirmation of the referrals.

Zimbabwe adopted and started implementing during the pilot the WHO recommendation of retesting all persons newly diagnosed as HIV positive with a second specimen before ART initiation to rule out potential misdiagnosis. ${ }^{16}$ Healthcare workers were trained on the retesting recommendation. The emphasis was for the retest to be conducted before ART initiation using the same testing algorithm, drawing another specimen on the same or different day, at the same or different testing site and by a different or same tester depending on staff availability. Systems to track and report cases of inconsistent results were put in place.

\section{Discussion and Lessons Learnt}

The created TWGs provided a platform for sharing experiences among implementing partners and MoHCC. This helped in the uniformity and standardising of communication messages, training material and implementation activities across the cascade of care in the pilot districts. ${ }^{17}$ Community knowledge, engagement and support were crucial elements for successfully implementing the guidelines. ${ }^{18,19}$ Health facilities preparedness was of significant importance. Despite resource constraints, many of the barriers and challenges were addressed by site-level actions using existing resources. Some of the activities did improve not only the quality and effectiveness of services across the cascade of care but also reduced the workload.

In the early stages of the implementation of Treat All, the focus was putting patients on antiretroviral therapy. Minimal attention was offered to other components of the cascade. With time, there was the realisation that 
Treat All is not just about ART initiation but needs to be implemented as a package. There was a need to call patients who had ART deferred due to eligibility criteria, intensify HIV testing, retesting before ART initiation, timely linkage to care of those identified HIV positives, adequate clinical and psychological readiness assessment before ART initiation. We then started to put in place strategies to address these other components of the package.

As the implementation of Treat All, progressed there was a decrease in the number of patients initiated on ART. Like with our experience, the upsurge and later the decrease was mainly due to exhaustion of the pool of patients who had ART deferred due to eligibility criteria. ${ }^{6,20}$ We had to put in place systems to track patients who had ART deferred because of eligibility criteria. The other reason that might have decreased patients starting ART was how HIV testing evolved during the pilot. When Treat All started, there was massive general community catch up testing. As we continued with that testing strategy, it became difficult to identify positives, and this pushed us towards starting targeted HIV testing both at facility and community levels.

With same-day ART initiation, the dilemma among healthcare workers was on how to compress the counselling sessions in a single day. Initially, it was a mammoth task, but surprisingly with time, most of the clinically ready patients could absorb the compressed sessions in a day and start treatment on the same day. This might have been due to the high acceptability of Treat All by the patients themselves. However, not all patients were started on ART on the same day. Early on, there was confusion in the meaning of Treat All (test and treat), with most healthcare workers thinking that all patients must be initiated on ART on the same-day of testing. The MoHCC later provided guidance, with the aim being to start patients on ART within 14 days after addressing all the critical psychological and clinical issues. ${ }^{9}$

Though the initiation of patients on ART regardless of clinical or immunological status had known benefits, the removal of the CD4 brought some practical implementation challenges. When the change in guidelines was announced, there was an abrupt decrease in funding for CD4 testing both for point of care and laboratorybased testing. ${ }^{21,22}$ The CD4 count has been used to identify patients with advanced disease. ${ }^{23}$ The process to identify these patients was now heavily relying on clinical assessment. The problem was further compounded by the fact that the sensitivity of clinical signs and symptoms in picking patients with advanced diseases is low. ${ }^{24,25}$ Initially, MoHCC and partners responded by capacitating healthcare workers in the clinical screening of common opportunistic infections, which might lead to immune reconstitution inflammatory syndrome in our setting, ie tuberculosis, cryptococcal meningitis and Kaposi sarcoma and later sourced consumables for CD4 testing.

The recommendation to retest all patients before ART initiation was based on published reports and unpublished programme data of HIV status misclassification, with false positive and false negative results. ${ }^{16,26,27}$ These reports raised concerns that some individuals might be starting on ART inappropriately. Treat All was accompanied by a massive scale-up of HIV testing services hence the need to put quality assurance measures. However, the problem of HIV testing misclassification in our setting might not be bad as initially thought. ${ }^{28,29}$

\section{Conclusion}

Coordination and well-structured engagement plans with the districts led to the successful implementation of the pilot. The created technical working groups provided standardisation and a platform for sharing experiences among implementing partners and MoHCC. Training and capacity building of healthcare workers through mentoring, support and supervision and provision of job aids was necessary for equipping the healthcare workers with the necessary skills. Community knowledge, engagement and support were also identified as crucial for the successful implementation of the guidelines. Site preparedness of the health facilities was significant. This enabled health facilities to develop mitigating strategies at the local level using existing resources.

Future research is required to understand Treat All implementation. Studies looking into how patient outcomes across the cascade of care (testing, linkage, ART initiation, adherence, retention and viral suppression) will evolve under Treat All are pertinent. With funding towards CD4 testing dwindling, there is a need to develop simpler clinical algorithms and point of care diagnostic tests to identify patients with advanced disease.

\section{Ethical Approval}

Not applicable. The manuscript is a report meant to share implementation experiences, and lessons learnt. It does not include individual patient-level data. 


\section{Acknowledgments}

The author was actively involved in the implementation of the HIV Treat All pilot during his stint at I-TECH, a PEPFAR partner that supported the pilot. He was part of the PEPFAR partners HIV Treat All Learning and documentation TWG. He appreciates the unwavering support he got from his colleagues from I-TECH, OPHID and MoHCC in coming up with the manuscript. He respects and acknowledges all the effort put by his colleagues in the preparation of various materials referred to in this article. He remains indebted to PEPFAR for funding and the MoHCC for supporting the pilot. Lastly, he appreciates all other partners, stakeholders, front line healthcare workers and PLHIV who made the pilot a success.

\section{Funding}

The HIV Treat All pilot was funded by PEPFAR through its two main implementation partners I-TECH and OPHID. Richard Makurumidze receives a PhD scholarship grant from the Institute of Tropical Medicine, funded by the Belgian Development Cooperation (DGD). The funders had no role in the preparation of the manuscript or decision to publish.

\section{Disclosure}

The author declares that he has no competing interests.

\section{References}

1. World Health Organisation (WHO). Consolidated guidelines on the use of antiretroviral drugs for treating and preventing HIV infection: recommendations for a Public Health Approach. Geneva, Switzerland; 2016. Available from: https://apps.who.int/iris/bitstream/handle/10665/208825/ 9789241549684_eng.pdf?sequence $=1$. Accessed August 3, 2021.

2. TEMPRANO ANRS 12136 Study Group. A trial of early antiretrovirals and isoniazid preventive therapy in Africa. $N$ Engl $J$ Med. 2015;373(9):808-822. doi:10.1056/NEJMoa1507198

3. Lundgren JD, Babiker AG; INSIGHT START Study Group. Initiation of antiretroviral therapy in early asymptomatic HIV infection. $N$ Engl J Med. 2015;373(9):795-807. doi:10.1056/NEJMoa1506816

4. United Nations Joint Programme on HIV/AIDS (UNAIDS). Understanding fast-track: Accelerating action to end the AIDS epidemic by 2030. 2015. Available from: https://www.unaids.org/sites/ default/files/media_asset/201506_JC2743_Understanding_\% 0AFastTrack_en.pdf.

5. World Health Organization (WHO). Progress Report 2016. Prevent HIV, Test and Treat All. Geneva, Switzerland; 2016. Available from: https:// apps.who.int/iris/handle/10665/251713. Accessed August 3, 2021.

6. Schwitters AM. Notes from the field: preliminary results after implementation of a universal treatment program (test and start) for persons living with HIV infection - lesotho, October 2015-February 2017. MMWR Morb Mortal Wkly Rep. 2017;66(30):813-814. doi:10.15585/ mmwr.mm6630a4

7. The Lancet. HIV: the question is not when to treat, but how to treat. Lancet. 2015;386(10002):1420. doi:10.1016/S0140-6736(15)00454-7
8. Ministry of Health and Child Care (MoHCC). Guidelines for antiretroviral therapy for the prevention and treatment of HIV in Zimbabwe, 2016; 2016. Available from: http://www.ophid.org/treatall-toolkit/MOHCCGuidelines/MOHCC_ARTGuidelines2016.pdf. Accessed August 3, 2021.

9. Ministry of Health and Child Care Zimbabwe (MoHCC). Operational and service delivery manual for the prevention, care and treatment of HIV in Zimbabwe; 2017. Available from: https://www.differentiated care.org/Portals/0/adam/Content/m2an155byU6RIoHeF4e4FQ/File/ MSFZimOSDMwebrevised.pdf. Accessed August 3, 2021.

10. Ministry of Health and Child Care (MoHCC). Consolidated HIV and AIDS job aide; 2017. Available from: http://differentiatedservicede livery.org/Portals/0/adam/Content/A8PhuO3FRUSJjWBADDpd1g/ File/MSFZimbabweJobAidewebversion-1.pdf. Accessed August 3, 2021.

11. Organisation for Public Health Interventions Development (OPHID). Treat all frequently asked questions; 2015. Available from: http:// www.ophid.org/sites/default/files/OPHIDTREATALLFAQ-min.pdf. Accessed August 3, 2021.

12. Organisation for Public Health Interventions Development (OPHID). HIV retesting: frequently asked questions; 2016. Available from: https://www.ophid.org/sites/default/files/OPHIDHIVRETESTFAQmin.pdf. Accessed August 3, 2021.

13. Organisation for Public Health Interventions Development (OPHID). Communication and Community Mobilisation- Raise Awareness and Gain Community Support for Treat All. 2016. Available from: http:// www.ophid.org/treat-all-toolkit/Tools/Too $1 \% 203 \% 20$ - \% 20Communication\%202017_FINAL.pdf.

14. Organisation for Public Health Interventions Development (OPHID). Site preparedness prepare your health facility for optimal implementation of treat all; 2016. Available from: http://www.ophid.org/treatall-toolkit/Tools/Tool2-SitePreparedness2017_FINAL_A4.pdf. Accessed August 3, 2021.

15. Talent Maphosa. Implementation \& impact of HIV treat All: big data, assessment trends and projections. IAS 2017 Conf; 2017. Available from: https://programme.ias2017.org/PAGMaterial/PPT/3021_4511/ ImplementationImpactofTreatAll_IAS2017_23Ju12017.pptx. Accessed August 3, 2021.

16. World Health Organization (WHO). WHO reminds national programmes to retest all newly diagnosed people with HIV. Available from: https://www.who.int/hiv/pub/vct/retest-newly-diagnosed-plhivfull/en/. Accessed May 10, 2021.

17. Principles for Digital Development (PDP). How to set up a technical working group how to set up a technical working group; 2014. Available from: https://digitalprinciples.org/wp-content/uploads/ PDD_HowTo_TechnicalWorkingGroup-v2.pdf. Accessed August 3, 2021.

18. Slevin KW, Ukpong M, Heise L. Community engagement in HIV prevention trials: evolution of the field and opportunities for growth; 2008:14. Available from: http://www.aids2031.org/pdfs/aids2031 pre confreading-communityengagement_west_pdf. Accessed August 3, 2021.

19. Grimsrud A, Wilkinson L, Eshun-Wilson I, Holmes C, Sikazwe I, Katz IT. Understanding engagement in HIV programmes: how health services can adapt to ensure no one is left behind. Curr HIV/AIDS Rep. 2020;17(5):458-466. doi:10.1007/s11904-020-00522-1

20. Mitruka K, Bamrotiya M, Agarwal R, et al. Implementation of the treat all policy among persons with HIV infection enrolled in care but not on antiretroviral therapy - India, May 2017-June 2018. Morb Mortal Wkly Rep. 2018;67(47):1305-1309. doi:10.15585/mmwr. mm6747a2

21. Balachandra S, Rogers JH, Ruangtragool L, et al. Concurrent advanced HIV disease and viral load suppression in a high-burden setting: findings from the 2015-6 ZIMPHIA survey. Yotebieng M, ed. PLoS One. 2020;15(6):e0230205. doi:10.1371/journal.pone.0230205 
22. Ford N, Meintjes G, Vitoria M, Greene G, Chiller T. The evolving role of CD4 cell counts in HIV care. Curr Opin HIV AIDS. 2017;12 (2):123-128. doi:10.1097/COH.0000000000000348

23. World Health Organization (WHO). Guidelines for managing advanced HIV disease and rapid initiation of antiretroviral therapy, July 2017; July, 2017. Available from: http://apps.who.int/iris/bit stream/10665/255884/1/9789241550062-eng.pdf?ua=1. Accessed August 3, 2021.

24. Munthali C, Taegtmeyer M, Garner PG, et al. Diagnostic accuracy of the WHO clinical staging system for defining eligibility for ART in sub-Saharan Africa: a systematic review and meta-analysis. J Int AIDS Soc. 2014;17(1):18932. doi:10.7448/IAS.17.1.18932

25. Baveewo S, Ssali F, Karamagi C, et al. Validation of World Health Organisation HIV/AIDS clinical staging in predicting initiation of antiretroviral therapy and clinical predictors of low CD4 cell count in Uganda. Semple MG, ed. PLoS One. 2011;6(5):e19089. doi:10.1371/ journal.pone. 0019089
26. ICAP at Columbia University. Handbook on Implementing HIV Retesting for Verification Before/At Antiretroviral Therapy Initiation; 2017.

27. Gonese E, Mushavi A, Mungati M, et al. Is Zimbabwe ready to transition from anonymous unlinked sero-surveillance to using prevention of mother to child transmission of HIV (PMTCT) program data for HIV surveillance?: results of PMTCT utility study, 2012. BMC Infect Dis. 2016;16(1):97. doi:10.1186/s12879-016-1425-2

28. Dupwa B, Kumar AMV, Tripathy JP, et al. Retesting for verification of HIV diagnosis before antiretroviral therapy initiation in Harare, Zimbabwe: is there a gap between policy and practice? Trans $R$ Soc Trop Med Hyg. 2019;113(10):610-616. doi:10.1093/trstmh/trz047

29. Gregson S, Moorhouse L, Dadirai T, et al. Comprehensive investigation of sources of misclassification errors in routine HIV testing in Zimbabwe. J Int AIDS Soc. 2021;24(4):1-10. doi:10.1002/jia2.25700

\section{Publish your work in this journal}

HIV/AIDS - Research and Palliative Care is an international, peerreviewed open-access journal focusing on advances in research in HIV, its clinical progression and management options including antiviral treatment, palliative care and public healthcare policies to control viral spread. The manuscript management system is completely online and includes a very quick and fair peer-review system, which is all easy to use. Visit http://www.dovepress.com/testimonials.php to read real quotes from published authors. 